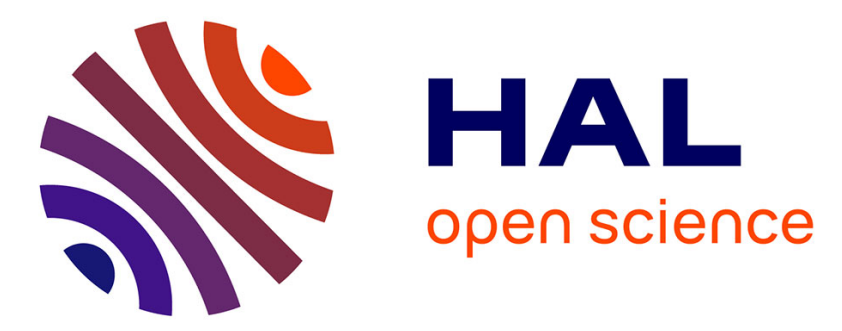

\title{
Construction d'une loi de fatigue à partir d'un modèle de forces cohésives: cas d'une fissure en mode III
}

\author{
Radhi Abdelmoula, Jean-Jacques Marigo, Thibaut Weller
}

\section{To cite this version:}

Radhi Abdelmoula, Jean-Jacques Marigo, Thibaut Weller. Construction d'une loi de fatigue à partir d'un modèle de forces cohésives: cas d'une fissure en mode III. Comptes Rendus Mécanique, 2009, 337, pp.53-59. 10.1016/j.crme.2008.12.001 . hal-00683019

\section{HAL Id: hal-00683019 https://hal.science/hal-00683019}

Submitted on 1 Dec 2021

HAL is a multi-disciplinary open access archive for the deposit and dissemination of scientific research documents, whether they are published or not. The documents may come from teaching and research institutions in France or abroad, or from public or private research centers.
L'archive ouverte pluridisciplinaire HAL, est destinée au dépôt et à la diffusion de documents scientifiques de niveau recherche, publiés ou non, émanant des établissements d'enseignement et de recherche français ou étrangers, des laboratoires publics ou privés.

\section{()ㅜ(1)}

Distributed under a Creative Commons Attribution - NonCommerciall 4.0 International 


\title{
Construction d'une loi de fatigue à partir d'un modèle de forces cohésives : cas d'une fissure en mode III
}

\author{
Radhi Abdelmoula ${ }^{\text {a }}$, Jean-Jacques Marigo ${ }^{\text {b,* }}$, Thibaut Weller ${ }^{c}$ \\ ${ }^{\text {a } L P M T M ~(U P R-C N R S ~ 9001), ~ U n i v e r s i t e ́ ~ P a r i s ~ X I I I, ~ a v e n u e ~ J .-B . ~ C l e m e n t, ~} 93430$ Villetaneuse, France \\ ${ }^{\mathrm{b}}$ Institut Jean le Rond d'Alembert (UMR 7190), Université Paris VI, 75252 Paris cedex 05, France \\ ${ }^{\mathrm{c}}$ LMGC (UMR 5508), Université Montpellier II, place Eugène-Bataillon, 34095 Montpellier cedex 5, France
}

\section{Résumé}

En partant d'un modèle de forces cohésives avec irréversibilité et en utilisant l'approche variationnelle de la rupture, nous considérons le problème de la propagation d'une fissure dans un milieu élastique bidimensionnel soumis à un chargement cyclique antiplan. Lorsque la longueur caractéristique du matériau est petite devant les dimensions de la structure, on se ramène grâce à un raisonnement à deux échelles à un problème local que l'on résout. On obtient ainsi une loi de fatigue reliant le taux d'avancée de la fissure à chaque cycle au facteur d'intensité des contraintes. En particulier on retrouve une loi de Paris avec la puissance 4 pour des faibles valeurs du facteur d'intensité des contraintes.

\begin{abstract}
Construction of a fatigue law from a cohesive force model: the mode III case. We consider the problem of crack propagation in a $2 \mathrm{~d}$ elastic medium submitted to an anti-plane cyclic loading, in the context of the variational approach to fracture with a cohesive forces model. When the size of the body is large compared to the material characteristic length, a twoscale method leads to a local problem whose solution is obtained in a closed form. We thus obtain a fatigue law where the growth of the crack length, at each cycle, is a function of the stress intensity factor. We recover, in particular, a Paris-type power law with the power 4 when the stress intensity factor is small.
\end{abstract}

Mots-clés : Fatigue; Mécanique de la rupture; Approche variationnelle; Analyse complexe

Keywords: Fatigue; Fracture mechanics; Variational approach; Complex analysis

\footnotetext{
* Auteur correspondant.

Adresses e-mail : radhi@galilee.univ-paris13.fr (R. Abdelmoula), marigo@lmm.jussieu.fr (J.-J. Marigo), weller@1mgc.univ-montp2.fr (T. Weller).
} 


\section{Abridged English version}

We consider a $2 \mathrm{~d}$ elastic body submitted to an anti-plane loading in which a crack propagates along a predefined crack path $\hat{\Gamma}$. The growth of the crack is governed by a cohesive force model of Dugdale-type in which we introduce the so-called cumulated tearing rate $\delta$ as an internal variable memorizing the history of the displacement jump $\llbracket u \rrbracket$ through $\hat{\Gamma}, \dot{\delta}=|\llbracket \dot{u} \rrbracket|$. The cohesive shear $\tau$ on $\hat{\Gamma}$ is obtained from the Dugdale surface energy density $\phi(\delta)=\min \left\{\tau_{c} \delta, G_{c}\right\}$, where $\tau_{c}$ is the critical shear, $G_{c}$ is the critical energy release rate and the ratio $\delta_{c}:=G_{c} / \tau_{c}$ is a material characteristic length. Specifically, $\tau=0$ on the part of $\hat{\Gamma}$ where $\delta>\delta_{c}$ whereas $\tau$ is given by (1) on the part of $\hat{\Gamma}$ where $\delta<\delta_{c}$. Following the variational approach to fracture [4] and considering a time-discretization, the evolution of the tearing is given by the sequence of minimization problems (2), (3). We assume that $\hat{\Gamma}$, identified with the segment $[0, L]$, can be divided at each step $i$ into three zones: the non-cohesive zone $\Gamma_{i}^{d}$, the cohesive zone $\Gamma_{i}^{c}$ and the sound zone $\Gamma_{i}^{b}$, cf. (4). We deduce from the minimization principle that $u_{i}, \delta_{i}, \ell_{i}$ and $\lambda_{i}$ must satisfy (5)-(7). In particular, (7) requires that the cumulated tearing rate is equal to $\delta_{c}$ at the tip of the non-cohesive zone and that there is no singularity at the tip of the cohesive zone. When the ratio $\delta_{c} / L$ is small, the problem can be investigated with a two-scale method. Indeed, in the case of a "symmetric" cyclic loading, the crack evolution enjoys the following properties: (i) at each cycle, the growth of the length of the non-cohesive zone is small; (ii) the length of the cohesive zone is always small; (iii) a quasi-stationary regime takes place in the sense that the evolution is approximately the same from a cycle to the next one. Therefore, a great number $N$ of cycles is needed so that the non-cohesive crack length be equal to $\ell$, with $\ell$ of the order of $L$. If we consider cycles close to $N$ and denote by $i$ the $i$ th half-cycle numbered from $N, i \in \mathbb{Z}$, the corresponding displacement field $u_{i}$ is in a first approximation independent of $i$, say $u$. This approximation $u$ is given by (8) and corresponds to the response of the body on a large scale, i.e. with a non-cohesive crack of length $\ell$ and without any cohesive zone. This field is singular at $\ell$, with a stress intensity factor $K$ depending on $\ell$ but not on $i$, see (9). But $u$ is a good approximation of $u_{i}$ only far from the crack tip. In the same manner, the growth rate of the crack length and the length of the cohesive zone are both small and independent of $i$ at each halfcycle $i$. They are denoted $\dot{\ell} / 2$ and $\lambda$ respectively. The rate $\dot{\ell}$ can be identified with the macroscopic rate $\mathrm{d} \ell / \mathrm{d} N$. The determination of $\dot{\ell}$ and $\lambda$ requires to obtain a more accurate approximation of $u_{i}$ near the crack tip. After a rescaling of the zone near the crack tip, the small scale problem giving the approximation $v_{i}$ of $u_{i}$ is set on an infinite domain with a semi-infinite straight crack along the axis $y=0$ where $(x, y)$ denotes the new coordinates system. By virtue of the quasi-stationarity property, the $v_{i}$ 's are related to $v_{0}$ by (10) and $v_{0}$ itself must satisfy (11), (12). Moreover $v_{0}$ must be non-singular at $x=\lambda$. This problem can be solved in a closed form with the help of the complex potentials theory [5]. Thus, $\lambda$ and the jump of $v_{0}$ through $y=0$ are given by (13)-(16). In (13), $G$ represents the potential energy release rate corresponding to a non-cohesive crack with length $\ell$. It is related to the stress intensity factor $K$ of $u$ by Irwin's formula. It remains to determine $\dot{\ell}$. From the optimal condition $\delta_{0}(0)=\delta_{c}$, we obtain by induction and by virtue of (10) that $\delta_{c}=\llbracket v_{0} \rrbracket(0)+2 \sum_{i=1}^{\infty} \llbracket v_{0} \rrbracket(i \dot{\ell} / 2)$. Using (15), we obtain Eq. (17) giving $\dot{\ell}$ in terms of $G$. This equation has no solution if $G<G_{c}$, an infinite number of solutions if $G=G_{c}$ and a unique solution if $0<G<G_{c}$, see (18). Moreover, when $G / G_{c}$ is small, the relation between $\dot{\ell}$ and $G$ is approximately a power law with the power 2 . In conclusion, we have obtained a propagation law which contains both Griffith and Paris laws. Griffith's law in the supercritical case where $G \geqslant G_{c}$ and Paris' law in the subcritical case where $G<G_{c}$. These results are similar to those previously obtained in [2] in a simplified one-dimensional context.

\section{Introduction}

Il a été annoncé dans [1] puis démontré dans [2], sur un problème modèle unidimensionnel de décollement de film mince, que l'on peut rendre compte de la fatigue, i.e. de la propagation de fissure sous chargement cyclique, à partir de modèles de forces cohésives de type Dugdale-Barenblatt, alors qu'on ne le peut pas à partir du modèle de Griffith. Il est toutefois essentiel pour cela d'introduire correctement la condition d'irréversibilité dans le modèle cohésif, dans l'esprit de ce qui avait été proposé dans [3]. En adoptant l'approche variationnelle de la rupture développée dans [4], l'évolution de la fissuration est alors régie par un problème incrémental de minimisation d'énergie. Le modèle cohésif contient nécessairement une longueur caractéristique du matériau. Lorsque cette longueur est petite devant les dimensions de la structure, la zone cohésive est également petite et on peut développer une méthode asymptotique rigoureuse pour obtenir une loi de fatigue «limite» en faisant tendre la longueur caractéristique vers 0 . Cette loi limite est une loi de type Paris mettant en relation le taux d'avancée de la longueur de la fissure à chaque cycle 
$\dot{\ell}:=\mathrm{d} \ell / \mathrm{d} N$ avec le taux de restitution d'énergie $G$, soit $\dot{\ell}=\mathrm{f}(G)$, la fonction $\mathrm{f}$ se déduisant de la loi cohésive. La construction de cette loi limite passe d'une part par la résolution d'un ou de plusieurs problèmes posés sur un domaine infini représentant le fond de fissure et d'autre part sur le concept de régime stationnaire disant que la zone cohésive et le champ de déplacement se reproduisent à l'identique à chaque cycle dans la translation $\dot{\ell}$. Tous ces résultats sont démontrés de façon rigoureuse dans le cas particulier [2] en s'appuyant de façon essentielle sur le caractère variationnel de l'approche. On se propose ici d'étendre la construction de la loi de fatigue limite dans le cas d'une fissure plane en mode III. Faute de place, nous ne détaillerons pas toutes les étapes, la démarche asymptotique sera seulement esquissée.

\section{Le modèle de forces cohésives et l'approche variationnelle}

On considère une structure cylindrique de section $\Omega$ soumise à un chargement anti-plan dépendant du temps (du type déplacement contrôlé sur une partie du bord, les forces volumiques étant nulles et les autres parties du bord étant libres) provoquant la propagation d'une fissure en mode III suivant un trajet connu a priori dont la trace sur $\Omega$ est une courbe $C^{1}$ notée $\hat{\Gamma}$. Le matériau constitutif est linéairement élastique de module de cisaillement $\mu$. Pour définir la loi de forces cohésives sur $\hat{\Gamma}$, on introduit tout d'abord une variable $\delta$ mémorisant l'histoire du saut de déplacement (antiplan) $\llbracket u \rrbracket$ à travers $\hat{\Gamma}$. On choisit ici le glissement cumulé défini par $\dot{\delta}=|\llbracket \dot{u} \rrbracket|$ avec la condition initiale $\left.\delta\right|_{t=0}=0$, le point désignant la dérivée par rapport au temps. On choisit ensuite comme densité surfacique d'énergie dissipée sur $\hat{\Gamma}$ celle correspondant au modèle de Dugdale, i.e. la fonction $\phi(\delta)=\min \left\{\tau_{c} \delta, G_{c}\right\}$ où $\tau_{c}$ est la contrainte de cisaillement critique et $G_{c}$ est le taux de restitution d'énergie critique. Le rapport $\delta_{c}:=G_{c} / \tau_{c}$ est le glissement critique, c'est une longueur caractéristique du matériau. Comme nous l'établissons dans (6), ces choix ont pour conséquence que la force cohésive de cisaillement $\tau$ sur $\hat{\Gamma}$ est nulle si $\delta>\delta_{c}$ (comme dans la théorie de Griffith), alors que, si $\delta \in\left[0, \delta_{c}\right), \tau$ est donnée par

$$
\tau=+\tau_{c} \quad \text { si } \llbracket \dot{u} \rrbracket>0, \quad \tau=-\tau_{c} \quad \text { si } \llbracket \dot{u} \rrbracket<0, \quad \tau \in\left[-\tau_{c},+\tau_{c}\right] \quad \text { si } \llbracket \dot{u} \rrbracket=0
$$

Pour déterminer l'évolution de la fissuration, il suffit de déterminer l'évolution de $u$ au cours du temps, l'évolution de son saut et du glissement cumulé s'en déduisant. On adopte l'approche variationnelle (quasistatique) développée dans [4] qui, après discrétisation en temps, se ramène à une séquence de problèmes de minimisation de l'énergie totale. De façon précise, ayant discrétisé l'histoire du chargement, on note $u_{i}$ le champ de déplacement dans $\Omega \backslash \hat{\Gamma}$, $\llbracket u_{i} \rrbracket$ et $\delta_{i}$ son saut et le glissement cumulé sur $\hat{\Gamma}$ au pas $i$. Si $\mathcal{C}_{i}$ désigne l'ensemble des déplacements admissibles au pas $i, u_{i-1}$ et $\delta_{i-1}$ étant supposés connus, $u_{i}$ et $\delta_{i}$ sont définis par

$$
\begin{aligned}
u_{i} & =\operatorname{Argmin}_{v \in \mathcal{C}_{i}}\left\{\int_{\Omega \backslash \hat{\Gamma}} \frac{\mu}{2} \nabla v \cdot \nabla v \mathrm{~d} x+\int_{\hat{\Gamma}} \phi\left(\delta_{i-1}+\left|\llbracket v-u_{i-1} \rrbracket\right|\right) \mathrm{d} s\right. \\
\delta_{i} & =\delta_{i-1}+\left|\llbracket u_{i}-u_{i-1} \rrbracket\right|
\end{aligned}
$$

C'est à partir de ce problème de minimisation incrémental (adapté au problème de décollement de film mince) que sont établies dans [2] toutes les propriétés de la loi de fatigue. Il est évidemment impossible de procéder de façon aussi déductive ici sans préciser la géométrie et le chargement. Faisons donc quelques hypothèses sur la structure de la solution. Au pas $i$, on suppose que l'on peut distinguer trois parties dans $\hat{\Gamma}$ : (i) une zone $\Gamma_{i}^{b}$, dite zone saine, où $\delta_{i}=0$, i.e. où aucun glissement relatif n'a encore eu lieu; (ii) une zone $\Gamma_{i}^{c}$, dite zone cohésive, où $0<\delta_{i}<\delta_{c}$; une zone $\Gamma_{i}^{d}$, dite zone non cohésive, où $\delta_{i}>\delta_{c}$. En paramétrant $\hat{\Gamma}$ par son abscisse curviligne $s \in[0, L]$ et en l'identifiant avec le segment $[0, L]$, supposons en outre que ces trois parties soient les segments suivants

$$
\Gamma_{i}^{d}=\left[0, \ell_{i}\right), \quad \Gamma_{i}^{c}=\left(\ell_{i}, \ell_{i}+\lambda_{i}\right), \quad \Gamma_{i}^{b}=\left[\ell_{i}+\lambda_{i}, L\right]
$$

$\ell_{i}$ et $\lambda_{i}$ représentant respectivement la position de la pointe non cohésive et la longueur de la zone cohésive. Du fait de l'irréversibilité, $\ell_{i-1} \leqslant \ell_{i}$ et $\ell_{i-1}+\lambda_{i-1} \leqslant \ell_{i}+\lambda_{i}$. En écrivant les conditions d'optimalité, on obtient que le minimiseur $u_{i}$ doit vérifier les équations d'équilibre et l'absence de forces cohésives sur $\Gamma_{i}^{d}$ :

$$
\Delta u_{i}=0 \quad \text { dans } \Omega \backslash \hat{\Gamma}, \quad \mu \frac{\partial u_{i}}{\partial n}=0 \quad \text { sur } \Gamma_{i}^{d}, \quad \llbracket u_{i} \rrbracket=0 \quad \text { sur } \Gamma_{i}^{b}
$$


Sur $\Gamma_{i}^{c}$, on distingue les zones $\Gamma_{i}^{c+}=\left\{s \in \Gamma_{i}^{c}: \llbracket u_{i} \rrbracket>\llbracket u_{i-1} \rrbracket\right\}$ et $\Gamma_{i}^{c-}=\left\{s \in \Gamma_{i}^{c}: \llbracket u_{i} \rrbracket<\llbracket u_{i-1} \rrbracket\right\}$ où le glissement relatif est actif, et la zone $\Gamma_{i}^{c 0}=\left\{s \in \Gamma_{i}^{c}: \llbracket u_{i} \rrbracket=\llbracket u_{i-1} \rrbracket\right\}$ où il ne l'est pas. On obtient alors

$$
\mu \frac{\partial u_{i}}{\partial n}=+\tau_{c} \quad \operatorname{sur} \Gamma_{i}^{c+}, \quad \mu \frac{\partial u_{i}}{\partial n}=-\tau_{c} \quad \operatorname{sur} \Gamma_{i}^{c-}, \quad \mu \frac{\partial u_{i}}{\partial n} \in\left[-\tau_{c},+\tau_{c}\right] \quad \operatorname{sur} \Gamma_{i}^{c 0}
$$

ce qui est conforme à (1). Notons que, même en supposant $\ell_{i}$ et $\lambda_{i}$ connus, le système (5), (6) est non linéaire en $u_{i}$ car les zones où le glissement est actif ne sont pas connues a priori. Cependant, dans le cas du modèle de Dugdale et en raisonnant à $\ell_{i}$ et $\lambda_{i}$ fixés, l'énergie totale est une fonction strictement convexe, ce qui assure l'unicité de $u_{i}$. Remarquons aussi que le champ $u_{i}$ ne peut être singulier qu'à la pointe $\ell_{i}+\lambda_{i}$, car les cisaillements sont bornés par $\tau_{c}$ sur $\Gamma_{i}^{d} \cup \Gamma_{i}^{c}$. La singularité, si elle existe, est une singularité classique en $K_{i}\left(\ell_{i}+\lambda_{i}\right) \sqrt{r} \sin \theta / 2$ avec $(r, \theta)$ les coordonnées polaires de pôle la pointe de la zone cohésive.

Une fois $u_{i}$ déterminé, l'énergie totale devient une fonction de $\ell_{i}$ et $\lambda_{i}$ qu'il reste à minimiser. En écrivant que la dérivée de l'énergie totale par rapport à $\ell_{i}$ est nulle, on obtient que le glissement cumulé à la pointe non cohésive est égal au glissement critique. En écrivant que la dérivée de l'énergie totale par rapport à $\lambda_{i}$ est nulle, on obtient que le champ $u_{i}$ ne doit pas être singulier à la pointe de la zone cohésive. En résumé, les deux équations donnant $\ell_{i}$ et $\lambda_{i}$ sont

$$
\delta_{i}\left(\ell_{i}\right)=\delta_{c}, \quad K_{i}\left(\ell_{i}+\lambda_{i}\right)=0
$$

Cependant, ce système étant non linéaire, on n'est pas assuré de l'unicité. Dans [2], on avait réussi à démontrer l'unicité en s'appuyant de façon essentielle sur le fait qu'on recherche un minimum, ce qui fournit des informations supplémentaires. On ne sait malheureusement pas prouver ici l'unicité dans le cas général.

\section{Cas d'une structure de grande taille sous chargement cyclique}

Supposons que la structure ait des dimensions grandes vis-à-vis de la longueur caractéristique du matériau, i.e. $\delta_{c} / L \ll 1$, et qu'elle soit soumise à un chargement cyclique «symétrique» dont l'intensité oscille entre $-U$ et $+U$. Dans le cas du décollement d'un film mince [2], on en déduisait les trois propriétés importantes suivantes :

(i) L'avancée de la pointe non cohésive à chaque cycle est petite devant les dimensions de la structure ;

(ii) La longueur de la zone cohésive est petite devant les dimensions de la structure;

(iii) D'un cycle au suivant, les champs varient peu, un régime quasi-stationnaire s'installe.

Nous les adoptons ici comme hypothèses de travail et vérifierons leur cohérence a posteriori. Elles permettent de faire une séparation d'échelles, aussi bien en espace qu'en temps.

Soit $\ell$ de l'ordre de $L$ et donc grand devant $\delta_{c}$. De (i) on déduit qu'il faut un nombre de cycles $N$ grand (d'autant plus grand que $\delta_{c} / L$ est petit) pour que la pointe non cohésive arrive en $\ell$. Envisageons les cycles voisins de $N$, i.e. les cycles $N+n$ avec $n \in\{0, \pm 1, \pm 2, \ldots\}$ indépendant de $\delta_{c} / L$. On peut distinguer deux échelles de nombre de cycles : la grande échelle $N$ et la petite échelle $n$. Intéressons-nous aux variations de la fissure à petite échelle autour de $N$. L'indice $i$ servira désormais à repérer les demi-cycles à partir de $N$, les indices pairs pour les fins de charge $(+U)$ et les indices impairs pour les fins de décharge $(-U)$.

De (i) et (iii) on déduit que l'avancée de la fissure à chaque demi-cycle autour de $N$ est petite et indépendante de $i$. (L'avancée est la même en charge et en décharge, du fait de la symétrie des cycles.) Notons-la $\dot{\ell} / 2$. Grâce à son indépendance par rapport à $i, \dot{\ell}$ s'identifie avec le taux d'avancée à grande échelle de la fissure $\mathrm{d} \ell / \mathrm{d} N$, cf. [4]. De plus, on déduit de (ii) que la longueur de la zone cohésive à la fin de chaque demi-cycle autour de $N$ est petite et indépendante de $i$. Notons-la $\lambda$. Par conséquent le déplacement $u_{2 i}$ est en première approximation égal à celui correspondant à une fissure de longueur $\ell$ sans zone cohésive, notons ce dernier $u$ (qui dépend donc de $N$ mais pas de $i$ ). De façon précise, $u$ vérifie le problème à grande échelle suivant (dans lequel il faut inclure la donnée à la limite $U$ sur $\partial \Omega)$ :

$$
\Delta u=0 \quad \text { dans } \Omega \backslash \hat{\Gamma}, \quad \mu \frac{\partial u}{\partial n}=0 \quad \text { sur }(0, \ell), \quad \llbracket u \rrbracket=0 \quad \operatorname{sur}[\ell, L]
$$


Du fait de la symétrie des cycles, $u_{2 i-1} \approx-u$. Ce champ $u$ est singulier à la pointe $\ell$ avec une singularité en $\sqrt{r}$ et un facteur d'intensité des contraintes $K$ (qui dépend de $\ell$ et est proportionnel à $U$ ) :

$$
u(\mathbf{x})=u\left(\mathbf{x}_{\ell}\right)+\frac{2 K}{\mu} \sqrt{\frac{r}{2 \pi}} \sin \frac{\theta}{2}+\text { partie régulière }
$$

où $u\left(\mathbf{x}_{\ell}\right)$ désigne le déplacement de la pointe de la fissure, $(r, \theta)$ les coordonnées polaires avec comme origine la pointe de la fissure et comme $\theta=0$ la direction de la tangente à $\hat{\Gamma}$ en $\ell$. Toutefois, $u$ ne sert d'approximation de $u_{i}$ qu'assez loin de la zone cohésive. Or on a besoin d'avoir une bonne approximation de $u_{i}$ au voisinage de la zone cohésive pour déterminer $\dot{\ell}$. Cela passe par la résolution d'un problème dit à petite échelle que nous présentons ci-dessous.

\section{Le problème en pointe de fissure}

On prend comme origine du repère la pointe $\ell$ de la zone non cohésive au cycle $N$, comme axe 1 la tangente à $\hat{\Gamma}$ en $\ell$ et on fait un changement d'échelle d'espace à l'aide d'un zoom. Le domaine à petite échelle est alors le plan $\mathbb{R}^{2}$ muni du système de coordonnées $(x, y)$. On note $v_{i}$ le champ de déplacement régnant dans ce domaine à la fin du demi-cycle $i$ qui servira d'approximation locale de $u_{i}-u\left(\mathbf{x}_{\ell}\right)$. Du fait de l'existence supposée d'un régime stationnaire à petite échelle de cycles (cf. la propriété (iii)) et du fait de la symétrie de chaque cycle, tous les $v_{i}$ se déduisent de $v_{0}$ par translation :

$$
v_{i}(x, y)=(-1)^{i} v_{0}(x-i \dot{\ell} / 2, y), \quad i \in \mathbb{Z}
$$

Il reste à déterminer $v_{0}, \lambda$ et $\dot{\ell}$. En $i=0$ la zone non cohésive $\gamma_{0}^{d}$ est le demi-axe $(-\infty, 0) \times\{0\}$ et la zone cohésive $\gamma_{0}^{c}$ le segment $[0, \lambda] \times\{0\}$. Le chargement à l'infini est celui de la singularité de $u$ (avec $K$ pour facteur d'intensité des contraintes). On suppose a priori que la zone cohésive est entièrement active, les forces cohésives valant $\tau_{c}$, et on vérifie a posteriori la cohérence de cette hypothèse. Cette vérification passe par le calcul du champ de déplacement $v_{t}$ à chaque instant $t \in[-1,0]$ de la montée en charge, mais ce calcul est trop long pour être reproduit ici. Le champ $v_{0}$ est donc tel que

$$
\Delta v_{0}=0 \quad \text { dans } \mathbb{R}^{2} \backslash\left(\gamma_{0}^{d} \cup \gamma_{0}^{c}\right), \quad \mu \frac{\partial v_{0}}{\partial y}= \begin{cases}0 & \text { sur } \gamma_{0}^{d} \\ +\tau_{c} & \text { sur } \gamma_{0}^{c}\end{cases}
$$

avec la condition à l'infini

$$
\lim _{r \rightarrow \infty}\left(v_{0}(x, y)-\frac{2 K}{\mu} \sqrt{\frac{r}{2 \pi}} \sin \frac{\theta}{2}\right)=0 \quad \text { où } x=r \cos \theta, y=r \sin \theta
$$

À $\lambda$ donné, ce problème admet une solution $v_{0}$ unique qui s'obtient explicitement à partir de la méthode des potentiels complexes, cf [5] :

$$
\mu \frac{\partial v_{0}}{\partial y}(x, y)+\mathbf{i} \mu \frac{\partial v_{0}}{\partial x}(x, y)=\psi(z)
$$

où $z=x+\mathbf{i} y, \mathbf{i}=\sqrt{-1}$ et $\psi$ est une fonction holomorphe dans le plan complexe privé de la demi-droite $\gamma_{0}^{d} \cup \gamma_{0}^{c}$, dont on déduit $v_{0}$ par intégration. La longueur de la zone cohésive $\lambda$ s'obtient en écrivant qu'il n'y a pas de singularité à la pointe cohésive, $\mathrm{cf}$. $(7)_{2}$. Finalement, on obtient

$$
\begin{aligned}
& \lambda=\frac{\pi}{4} \frac{G}{G_{c}} \frac{\mu}{\tau_{c}} \delta_{c} \quad \text { avec } G=\frac{K^{2}}{2 \mu} \\
& \psi(z)=\tau_{c}+\frac{\mathbf{i} \tau_{c}}{\pi}(\log (\sqrt{\lambda}+\mathbf{i} \sqrt{z-\lambda})-\log (\sqrt{\lambda}-\mathbf{i} \sqrt{z-\lambda}))
\end{aligned}
$$

Dans (13), $G$ représente le taux de restitution d'énergie potentielle associé à la structure comportant une fissure non cohésive de longueur $\ell$, cf le problème à grande échelle (8). Il est relié à $K$ par la formule d'Irwin. Dans (14), Log désigne la détermination principale du logarithme complexe. On en déduit que le cisaillement et le saut de $v_{0}$ sont donnés par

$$
\mu \frac{\partial v_{0}}{\partial y}(x, 0)=\left(1-\frac{2}{\pi} \operatorname{Arcsin} \sqrt{1-\frac{\lambda}{x}}\right) \tau_{c}, \quad x>\lambda ; \quad \llbracket v_{0} \rrbracket(x)=V\left(\frac{x}{\lambda}\right) \frac{G}{G_{c}} \delta_{c}, \quad x \geqslant 0
$$


où $V$ désigne la fonction définie sur $\mathbb{R}_{*}^{+}$par

$$
V(l)= \begin{cases}\sqrt{1-l}+l \ln (1-\sqrt{1-l})-l \ln \sqrt{l} & \text { si } 0<l \leqslant 1 \\ 0 & \text { si } l \geqslant 1\end{cases}
$$

et prolongée par continuité par 1 en 0 . (La valeur du saut pour $x<0$ n'est pas donné ici, étant inutile pour la suite.)

\section{La loi de fatigue}

Pour obtenir $\dot{\ell}$ on écrit, conformément à $(7)_{1}, \delta_{0}(0)=\delta_{c}$. À partir du calcul de $v_{t}$ pour $t \in[0,1]$, on montre que $\llbracket \dot{v}_{t} \rrbracket \geqslant 0$, ce qui permet d'écrire $\delta_{0}(0)=\delta_{-1}(0)+v_{0}(0)-v_{-1}(0)$. Grâce à (10) et en procédant par induction, on obtient $\delta_{c}=\llbracket v_{0} \rrbracket(0)+2 \sum_{i=1}^{\infty} \llbracket v_{0} \rrbracket(i \dot{\ell} / 2)$ qui, grâce à (13) et (15), fournit l'équation cherchée en $\dot{\ell}$ :

$$
1-\frac{G}{G_{c}}={\frac{G}{G_{c}}}_{i=1}^{\infty} 2 V\left(\frac{i \dot{\ell}}{2 \lambda}\right)
$$

Dans (17), la série comporte en fait un nombre fini de termes dès que $\dot{\ell}>0$ car tous les termes s'annulent à partir d'un certain rang $n=n(\dot{\ell})$. En étudiant cette équation en $\dot{\ell}$ à $G$ fixé, on obtient les résultats suivants

(i) Si $G>G_{c}$, alors il n'y a pas de solution;

(ii) Si $G=G_{c}$, alors tout $\dot{\ell} \geqslant \frac{\pi}{2} \frac{\mu}{\tau_{c}} \delta_{c}$ est solution;

(iii) Si $0<G<G_{c}$, alors il existe une seule solution qui peut s'écrire

$$
\dot{\ell}=\frac{\pi}{2} \frac{\mu}{\tau_{c}} f\left(\frac{G}{G_{c}}\right) \delta_{c}
$$

$g \mapsto \mathrm{f}(g)$ étant une fonction (sans dimension) de classe $C^{1}$, strictement croissante de 0 à 1 quand $g$ croît de 0 à 1. (On ne peut avoir accès à f que numériquement.)

(iv) Pour des faibles valeurs de $g=G / G_{c}$, on a $\mathrm{f}(g)=2 g^{2} / 3+\mathrm{o}\left(g^{2}\right)$ et on obtient la loi puissance

$$
\dot{\ell} \approx \frac{\pi}{3} \frac{\mu}{\tau_{c}}\left(\frac{G}{G_{c}}\right)^{2} \delta_{c}
$$

Autrement dit, la loi de fatigue obtenue contient la loi de Griffith puisqu'elle interdit des valeurs de $G>G_{c}$ et qu'elle laisse indéterminée $\dot{\ell}$ quand $G=G_{c}$. En régime sous-critique, i.e. quand $G<G_{c}$, elle constitue une loi de type Paris, le taux d'avancée de la fissure étant fonction du taux de restitution d'énergie. Elle s'apparente à une loi puissance en $G^{2}$ et donc en $K^{4}$ pour des faibles valeurs de $G / G_{c}$. Tous ces résultats sont en accord avec ce qui avait été établi dans [2] dans le cas unidimensionnel du décollement d'un film mince inextensible. La loi de Paris obtenue y était évidemment différente (en particulier la puissance était $3 / 2$ et non 2). La puissance 2 trouvée ici est due à la fois au fait que l'on est en 2D et que le comportement du matériau est élastique linéaire.

Il reste à vérifier a posteriori la cohérence des hypothèses de travail adoptées dans la Section 3. On voit qu'elles sont toutes satisfaites pourvu que l'on reste toujours en régime sous-critique. En effet tant que $G<G_{c}$, $\dot{\ell}$ et $\lambda$ sont de l'ordre de $\delta_{c}$, donc petits devant $L$, et on a construit une solution stationnaire du problème à petite échelle. Le fait que $G<G_{c}$ est du ressort du problème à grande échelle donnant $u$ en fonction de $\ell$ et de $U$. Mais comme $G$ est proportionnel à $U^{2}$, il suffit donc que l'amplitude du chargement cyclique soit suffisamment faible pour que l'on soit en régime sous-critique.

\section{Conclusion et perspectives}

Si l'on admet les trois propriétés présentées dans la Section 3, conséquences de la petitesse de la longueur caractéristique par rapport aux dimensions de la structure, on voit que la construction de la loi de fatigue repose essentiellement sur la résolution du problème à petite échelle consacré à l'étude des champs au voisinage de la zone cohésive. La loi de fatigue obtenue dépend donc de la loi cohésive adoptée, du fait que l'on soit en mode III et que les cycles soient symétriques. Mais elle ne dépend ni de la géométrie de la structure, ni de la façon dont on applique le chargement, ni de la forme générale de la fissure (ni de la présence d'autres fissures). On pourrait suivre la même 
démarche en changeant de loi cohésive, de mode ou de forme de cycle (au prix d'une complexification des calculs qui pourrait exiger le recours au numérique). Il s'agirait de voir alors en quoi la loi obtenue dépend de ces différents paramètres mécaniques.

\section{Références}

[1] A. Jaubert, J.-J. Marigo, L'approche variationnelle de la fatigue : des premiers résultats, C. R. Mecanique 333 (7) (2005) $550-556$.

[2] A. Jaubert, J.-J. Marigo, Justification of Paris-type fatigue laws from cohesive forces model via a variational approach, Continuum Mech. Thermodynam. 18 (1) (2006) 23-45.

[3] J.-J. Marigo, Modelling of brittle and fatigue damage for elastic material by growth of microvoids, Engrg. Fracture Mech. 21 (4) (1985) 861-874.

[4] B. Bourdin, G.A. Francfort, J.-J. Marigo, The variational approach to fracture, J. Elasticity 91 (1-3) (2008) 5-148.

[5] N.I. Mushkelishvili, Some Basic Problems in the Mathematical Theory of Elasticity, Noordhoff, 1953. 